\title{
Quantum-Classical Correspondence for the Equilibrium Distributions of Two Interacting Spins
}

\author{
J. Emerson and L.E. Ballentine \\ Physics Department, Simon Fraser University, Burnaby, British Columbia, Canada V5A 1S6
}

(November 6, 2018)

\begin{abstract}
We consider the quantum and classical Liouville dynamics of a non-integrable model of two coupled spins. Initially localised quantum states spread exponentially to the system dimension when the classical dynamics are chaotic. The long-time behaviour of the quantum probability distributions and, in particular, the parameter-dependent rates of relaxation to the equilibrium state are surprisingly well approximated by the classical Liouville mechanics even for small quantum numbers. As the accessible classical phase space becomes predominantly chaotic, the classical and quantum probability equilibrium configurations approach the microcanonical distribution, although the quantum equilibrium distributions exhibit characteristic 'minimum' fluctuations away from the microcanonical state. The magnitudes of the quantum-classical differences arising from the equilibrium quantum fluctuations are studied for both pure and mixed (dynamically entangled) quantum states. In both cases the standard deviation of these fluctuations decreases as $(\hbar / \mathcal{J})^{1 / 2}$, where $\mathcal{J}$ is a measure of the system size. In conclusion, under a variety of conditions the differences between quantum and classical Liouville mechanics are shown to become vanishingly small in the classical limit $(\mathcal{J} / \hbar \rightarrow \infty)$ of a non-dissipative model endowed with only a few degrees of freedom.
\end{abstract}

05.45.MT,03.65.TA,03.65.Yz

\section{INTRODUCTION}

The study of chaos in quantum dynamics has led to differing views on the conditions required for demonstrating quantum-classical correspondence [1,2]. Moreover, the criteria by which this correspondence should be measured have also been a subject of some controversy [3 5]. While much of the earlier work on this topic is concerned with characterizing the degree of correspondence between quantum expectation values and classical dynamical variables [6] 8], the more recent approach is to focus on differences between the properties of quantum states and associated classical phase space densities evolved according to Liouville's equation [1] 9 [13].

Several authors have examined quantum-classical correspondence by considering the effects of interactions with a stochastic environment [14 16], a process sometimes called decoherence. While this process may improve the degree of quantum-classical correspondence for fixed quantum numbers, it has been further suggested that the limit of large quantum numbers is inadequate for correspondence, and that decoherence must be taken into account to generate classical appearances from quantum theory; this view has been argued to apply even in the case of macroscopic bodies that are described initially by well-localised states, provided their classical motion is chaotic [5, 17, 18]. In this paper we examine how the degree of correspondence with Liouville dynamics scales specifically in the limit of large quantum numbers. This "classical limit" is distinct from a "thermodynamic limit", that is, a limit involving many quantum numbers.

The degree of Liouville correspondence has been characterized previously by studying the differences between the means and variances of the dynamical variables [1,9, 11, 13, 19. This involves a comparison of quantum expectation values and classical ensemble averages. However, these low-order moments give only crude information about the differences between the quantum and classical states. Specifically, the quantum state may exhibit coarse structure which differs significantly from the classical state although the means and variances (for some simple observabes) are nearly the same for the quantum and classical states. Moreover, much of the previous work was concerned with correspondence at early times, or more precisely, in the Ehrenfest regime when the states are narrow compared to system dimensions [12,13].

Another approach is to identify quantum-classical differences with differences between the Wigner quasi-distribution and the classical phase space density [2]. This approach is objectionable because the Wigner quasi-distribution may take on negative values and therefore may not be interpreted as a "classically observable" phase space distribution. It is possible to consider instead smoothed quantum phase space distributions, but in this case the residual quantumclassical differences still do not have clear experimental significance.

In this paper, we characterize the degree of quantum-classical correspondence by comparing quantum probability distributions for dynamical variables with the corresponding classical marginal distributions for these dynamical 
variables. These are well-defined classical observables that describe the distribution of outcomes upon measurement of the given dynamical variable. We are interested in the differences that arise on a fine scale and therefore characterize the typical quantum-classical deviations that arise in bins of width $\hbar$.

The dynamics are generated by a model of interacting spins that we have studied previously in Ref. [13]. The Hilbert space is finite dimensional so no artificial truncation of the state is required. The quantum time-evolution is unitary and the classical motion is volume-preserving (symplectic). In the case of classically chaotic motion, we follow initially localised states until they have evolved well beyond the relaxation time-scale of the classical density. Throughout the paper we emphasize that the quantum signatures of chaos that appear in the quantum distributions are the same as those that appear in the marginal classical distributions. In particular, the quantum relaxation rates can be accurately estimated from the Liouville dynamics of an approximately matching initial phase space density. This purely classical approximation is surprisingly accurate even for small quantum numbers, but may be most useful for the theoretical description of mesoscopic systems since the purely classical calculations do not scale with the quantum numbers.

The quantum and classical probability distributions remain close even after the states have spread to the system dimension. Specifically, in mixed regimes, the quantum distributions exhibit an equilibrium shape that reflects the details of the classical KAM surfaces. When the classical manifold is predominantly chaotic, the quantum and classical states relax close to the microcanonical state. However, in both of these chaotic regimes the equilibrium quantum distributions exhibit characteristic fluctuations away from the classical ones. We demonstrate that the standard deviation of these quantum-classical differences becomes vanishingly small in the classical limit, $\mathcal{J} / \hbar \rightarrow \infty$, where $\mathcal{J}$ is a characteristic system dimension.

This paper is organised as follows. In section II we describe the quantum and classical models for our system. In section III we describe the initial quantum states and corresponding classical densities. In section IV we give an overview of the dynamics of the probability distributions in the different classical regimes. In section $\mathrm{V}$ we examine the quantum and classical relaxation rates using the Shannon entropy. In section VI we give an overview of the time-development of the small differences between the quantum and classical probability distributions. In section VII we show that the relative quantum-classical differences decrease as an inverse power of the Hilbert space dimension. In section VIII we provide a brief summary and explain how our results inform current discussions of the necessary and sufficient conditions for the emergence of classical properties from quantum mechanics.

\section{THE MODEL}

We consider the quantum and classical dynamics generated by a non-integrable model of two interacting spins,

$$
H=a\left(S_{z}+L_{z}\right)+c S_{x} L_{x} \sum_{n=-\infty}^{\infty} \delta(t-n)
$$

where $\mathbf{S}=\left(S_{x}, S_{y}, S_{z}\right)$ and $\mathbf{L}=\left(L_{x}, L_{y}, L_{z}\right)$. The first two terms correspond to simple rotation of both spins about the $z$-axis through an angle $a$ with range $2 \pi$ radians. The sum over coupling terms describes an infinite sequence of $\delta$-function interactions at times $t=n$ for integer $n$. Each interaction term produces an impulsive rotation of each spin about the $x$-axis by an angle proportional to the $x$-component of the other spin.

\section{A. The Quantum Dynamics}

To obtain the quantum dynamics we interpret the Cartesian components of the spins as operators satisfying the usual angular momentum commutation relations,

$$
\begin{aligned}
& {\left[S_{i}, S_{j}\right]=i \epsilon_{i j k} S_{k}} \\
& {\left[L_{i}, L_{j}\right]=i \epsilon_{i j k} L_{k}} \\
& {\left[J_{i}, J_{j}\right]=i \epsilon_{i j k} J_{k} .}
\end{aligned}
$$

In the above we have set $\hbar=1$ and introduced the total angular momentum vector $\mathbf{J}=\mathbf{S}+\mathbf{L}$.

The Hamiltonian (11) possesses kinematic constants of the motion, $\left[\mathbf{S}^{2}, H\right]=0$ and $\left[\mathbf{L}^{2}, H\right]=0$. Thus the total state vector $|\psi\rangle$ can be represented in an invariant Hilbert space $\mathcal{H}=\mathcal{H}_{s} \otimes \mathcal{H}_{l}$, with dimension $N=(2 s+1) \times(2 l+1)$, that is spanned by the orthonormal vectors

$$
\left|s, l, m_{s}, m_{l}\right\rangle=\left|s, m_{s}\right\rangle \otimes\left|l, m_{l}\right\rangle
$$


with $m_{s} \in\{s, s-1, \ldots,-s\}$ and $m_{l} \in\{l, l-1, \ldots,-l\}$.

It should be noted that the components of the total angular momentum are not conserved $\left[J_{i}, H\right] \neq 0$. The $z$ component is subject to the selection rule, $\Delta J_{z}=\{ \pm 2,0\}$, and consequently the full Hilbert space can be decomposed into two invariant subspaces.

The periodic sequence of interactions introduced by the $\delta$-function produces a quantum mapping. The timeevolution for a single iteration, from just before a kick to just before the next, is produced by the unitary transformation,

$$
|\psi(n+1)\rangle=F|\psi(n)\rangle,
$$

where $F$ is the single-step Floquet operator,

$$
F=\exp \left[-i a\left(S_{z}+L_{z}\right)\right] \exp \left[-i c S_{x} \otimes L_{x}\right] .
$$

The quantum dynamics are thus specified by two parameters, $a$ and $c$, and two quantum numbers, $s$ and $l$.

\section{B. Classical Map}

For the Hamiltonian (11) the corresponding classical equations of motion are obtained by interpreting the angular momentum components as dynamical variables satisfying,

$$
\begin{aligned}
& \left\{S_{i}, S_{j}\right\}=\epsilon_{i j k} S_{k} \\
& \left\{L_{i}, L_{j}\right\}=\epsilon_{i j k} L_{k} \\
& \left\{J_{i}, J_{j}\right\}=\epsilon_{i j k} J_{k},
\end{aligned}
$$

with $\{\cdot, \cdot\}$ denoting the Poisson bracket. The periodic $\delta$-function in the coupling term can be used to reduce the time-evolution to a stroboscopic mapping at times $t=n$, for integer $n$,

$$
\begin{aligned}
\tilde{S}_{x}^{n+1} & =\tilde{S}_{x}^{n} \cos (a)-\left[\tilde{S}_{y}^{n} \cos \left(\gamma r \tilde{L}_{x}^{n}\right)-\tilde{S}_{z}^{n} \sin \left(\gamma r \tilde{L}_{x}^{n}\right)\right] \sin (a), \\
\tilde{S}_{y}^{n+1} & =\left[\tilde{S}_{y}^{n} \cos \left(\gamma r \tilde{L}_{x}^{n}\right)-\tilde{S}_{z}^{n} \sin \left(\gamma r \tilde{L}_{x}^{n}\right)\right] \cos (a)+\tilde{S}_{x}^{n} \sin (a), \\
\tilde{S}_{z}^{n+1} & =\tilde{S}_{z}^{n} \cos \left(\gamma r \tilde{L}_{x}^{n}\right)+\tilde{S}_{y}^{n} \sin \left(\gamma r \tilde{L}_{x}^{n}\right) \\
\tilde{L}_{x}^{n+1} & =\tilde{L}_{x}^{n} \cos (a)-\left[\tilde{L}_{y}^{n} \cos \left(\gamma \tilde{S}_{x}^{n}\right)-\tilde{L}_{z}^{n} \sin \left(\gamma \tilde{S}_{x}^{n}\right)\right] \sin (a), \\
\tilde{L}_{y}^{n+1} & =\left[\tilde{L}_{y}^{n} \cos \left(\gamma \tilde{S}_{x}^{n}\right)-\tilde{L}_{z}^{n} \sin \left(\gamma \tilde{S}_{x}^{n}\right)\right] \cos (a)+\tilde{L}_{x}^{n} \sin (a), \\
\tilde{L}_{z}^{n+1} & =\tilde{L}_{z}^{n} \cos \left(\gamma \tilde{S}_{x}^{n}\right)+\tilde{L}_{y}^{n} \sin \left(\gamma \tilde{S}_{x}^{n}\right) .
\end{aligned}
$$

Here $\tilde{\mathbf{L}}=\mathbf{L} /|\mathbf{L}|, \tilde{\mathbf{S}}=\mathbf{S} /|\mathbf{S}|$, and we have introduced the parameters $\gamma=c|\mathbf{S}|$ and $r=|\mathbf{L}| /|\mathbf{S}|$. The mapping equations (5) describe the time-evolution of (11) from just before one kick to just before the next.

Since the magnitudes of both spins are conserved, $\left\{\mathbf{S}^{2}, H\right\}=\left\{\mathbf{L}^{2}, H\right\}=0$, the stroboscopic motion is actually confined to the four-dimensional manifold $\mathcal{P}=\mathcal{S}^{2} \times \mathcal{S}^{2}$, which corresponds to the surfaces of two spheres. This is manifest when the mapping (5) is expressed in terms of the four canonical coordinates $\mathbf{x}=\left(S_{z}, \phi_{s}, L_{z}, \phi_{l}\right)$, where $\phi_{s}=\tan \left(S_{y} / S_{x}\right)$ and $\phi_{l}=\tan \left(L_{y} / L_{x}\right)$. We will refer to the mapping (5) in canonical form using the shorthand notation $\mathbf{x}^{n+1}=\mathbf{F}\left(\mathbf{x}^{n}\right)$. It is also useful to introduce a complete set of spherical coordinates $\vec{\theta}=\left(\theta_{s}, \phi_{s}, \theta_{l}, \phi_{l}\right)$ where $\theta_{s}=\cos ^{-1}\left(S_{z} /|\mathbf{S}|\right)$ and $\theta_{l}=\cos ^{-1}\left(L_{z} /|\mathbf{L}|\right)$.

As in the quantum model, the components of total angular momentum are not constants of the motion $\left\{J_{i}, H\right\} \neq 0$. On the other hand, the quantum selection rule $\Delta J_{z}=\{ \pm 2,0\}$ has no classical analogue.

The mapping (5) on the reduced surface $\mathcal{P}$ enjoys a rather large parameter space. The dynamics are determined from three independent dimensionless parameters $(a, \gamma$, and $r \geq 1)$, where $\gamma=c|\mathbf{S}|$ is a dimensionless coupling strength and $r=|\mathbf{L}| /|\mathbf{S}|$ corresponds to the ratio of the magnitudes of the two spins. The dependence of the classical behaviour on these parameters is described in Ref. [13]. 


\section{The Liouville Dynamics}

We are interested in comparing the quantum dynamics generated by (3) with the corresponding Liouville dynamics of a classical distribution. The time-evolution of a Liouville density is generated by the partial differential equation,

$$
\frac{\partial \rho_{c}(\mathbf{x}, t)}{\partial t}=-\left\{\rho_{c}, H\right\}
$$

where $H$ stands for the Hamiltonian (11) and $\mathbf{x}=\left(S_{z}, \phi_{s}, L_{z}, \phi_{l}\right)$.

The solution to (6) can be expressed in the compact form,

$$
\rho_{c}(\mathbf{x}, t)=\int_{\mathcal{P}} \mathrm{d} \mu(\mathbf{y}) \delta(\mathbf{x}-\mathbf{x}(t, \mathbf{y})) \rho_{c}(\mathbf{y}, 0),
$$

with measure,

$$
d \mu(\mathbf{y})=d \tilde{S}_{z} d \phi_{s} d \tilde{L}_{z} d \phi_{l}
$$

and where the time-dependent functions $\mathbf{x}(t, \mathbf{y}) \in \mathcal{P}$ are solutions to the equations of motion (5) with initial conditions $\mathbf{y} \in \mathcal{P}$. This solution expresses the fact that Liouville's equation (6) describes the dynamics of a classical density of points evolving in phase space under the Hamiltonian flow. We exploit this fact to numerically solve (6) by randomly generating initial conditions consistent with an initial phase space distribution $\rho_{c}(\mathbf{x}, 0)$ and then time-evolving each of these initial conditions using the equations of motion (5).

\section{Correspondence Between Quantum and Classical Models}

For a quantum system specified by the four numbers $\{a, c, s, l\}$ we determine the corresponding classical parameters $\{a, \gamma, r\}$ by first defining the classical magnitudes in terms of the quantum magnitudes,

$$
\begin{aligned}
& |\mathbf{S}|=\sqrt{s(s+1)} \\
& |\mathbf{L}|=\sqrt{l(l+1)},
\end{aligned}
$$

where the quantities on the left hand side are the lengths of the classical spins and those on the right are the quantum numbers. If we set the Hamiltonian coefficients $a$ and $c$ numerically equal for both models, then the remaining two dimensionless classical parameters are determined,

$$
\begin{gathered}
r=\sqrt{\frac{l(l+1)}{s(s+1)}} \\
\gamma=c \sqrt{s(s+1)} .
\end{gathered}
$$

We are interested in extrapolating the behaviour of the quantum dynamics in the limit $s \rightarrow \infty$ and $l \rightarrow \infty$. This is accomplished by studying sequences of quantum models with increasing $s$ and $l$ chosen so that $r$ and $\gamma$ are held fixed. Since $s$ and $l$ are restricted to integer (or half-integer) values, the corresponding classical $r$ will actually vary slightly for each member of this sequence (although $\gamma$ can be matched exactly by varying the quantum parameter $c$ slightly). In the limit $s \rightarrow \infty$ and $l \rightarrow \infty$ this variation becomes increasingly small since $r=\sqrt{l(l+1) / s(s+1)} \rightarrow l / s$. We have examined the effect of the small variations in the value of $r$ on the classical behaviour and found this variation to have negligible effect.

\section{INITIAL STATES}

We consider initial quantum states which are pure and separable,

$$
|\psi(0)\rangle=\left|\psi_{s}(0)\right\rangle \otimes\left|\psi_{l}(0)\right\rangle .
$$

The initial state of each subsystem is a directed angular momentum state, 


$$
|\theta, \phi\rangle=R^{(j)}(\theta, \phi)|j, j\rangle,
$$

where $j$ in this section refers to either $l$ or $s$. This is a localised state, i.e. one of maximum polarization in the direction $(\theta, \phi)$, with expectation values of the spin components confined to the surface of a 2 -sphere,

$$
\begin{aligned}
\left\langle\theta, \phi\left|J_{z}\right| \theta, \phi\right\rangle & =j \cos \theta \\
\left\langle\theta, \phi\left|J_{x} \pm i J_{y}\right| \theta, \phi\right\rangle & =j e^{ \pm i \phi} \sin \theta .
\end{aligned}
$$

The states (12) are the SU(2) coherent states, which, like their counterparts in the Euclidean phase space, are minimum uncertainty states [20]; the normalized variance of the quadratic operator,

$$
\Delta \tilde{\mathbf{J}}^{2}=\frac{\left\langle\theta, \phi\left|\mathbf{J}^{2}\right| \theta, \phi\right\rangle-\langle\theta, \phi|\mathbf{J}| \theta, \phi\rangle^{2}}{j(j+1)}=\frac{1}{(j+1)},
$$

is minimised for given $j$ and vanishes in the limit $j \rightarrow \infty$. The coherent states directed along the $z$-axis, $|j, j\rangle$ and $|j,-j\rangle$, saturate the inequality of the Heisenberg uncertainty relation,

$$
\left\langle J_{x}^{2}\right\rangle\left\langle J_{y}^{2}\right\rangle \geq \frac{\left\langle J_{z}\right\rangle^{2}}{4}
$$

although this inequality is not saturated for coherent states polarized in other directions.

We would like to construct a classical Liouville density on the 2-sphere with marginal distributions that match the quantum probability distributions. But we have shown previously that this is impossible for the $\mathrm{SU}(2)$ coherent states [13]. Thus from the outset it is clear that any choice of initial classical state will exhibit residual discrepancy in matching some of the initial quantum moments.

We have examined the correspondence properties of several different classical distributions. These included the vector model distribution described in the Appendix of [13] and the Gaussian distribution used by Fox and Elston in correspondence studies of the kicked top $[10]$. We selected the density,

$$
\rho_{c}(\theta, \phi) \sin \theta d \theta d \phi=C \exp \left[-\frac{\left.2 \sin ^{2}\left(\frac{\theta}{2}\right)\right)}{\sigma^{2}}\right] \sin \theta d \theta d \phi
$$

with $C=\left[2 \pi \sigma^{2}\left(1-\exp \left(-2 \sigma^{-2}\right)\right)\right]^{-1}$, instead of those previously considered because it is periodic under $2 \pi$ rotation. The classical density (16) has a maximum along the $+z$-axis, corresponding to the coherent state $|j, j\rangle$. An initial state directed along $\left(\theta_{o}, \phi_{o}\right)$ is produced by a rigid body rotation of $(16)$ by an angle $\theta_{o}$ about the $y$-axis followed by rotation through an angle $\phi_{o}$ about the $z$-axis. The variance $\sigma^{2}$ is a free parameter of the distribution. Although $\sigma^{2}$ cannot be chosen so that all low order moments are satisfied, the choice $\sigma^{-2}=2|\mathbf{J}|$, where $|\mathbf{J}|^{2}=j(j+1)$, produces a reasonable compromise, as discussed in [13].

\section{DYNAMICAL BEHAVIOUR OF PROBABILITY DISTRIBUTIONS}

In the case of a mixing classical system, initial densities with non-zero measure are expected to spread in an increasingly uniform manner throughout the accessible phase space. The term uniform is meant to apply specifcally in a coarse-grained sense. For some simple maps, such as the baker's map, it is possible to show that this rate of relaxation to the equilibrium configuration occurs exponentially with time [21].

The spin map we consider (5) is not mixing on the accessible classical manifold $\mathcal{P}$, but has mixed dynamics: depending on the system parameters, the surface $\mathcal{P}$ can generally be decomposed into regions of regular dynamics and a connected region of chaotic dynamics [13]. In parameter regimes that are predominantly chaotic, we expect behaviour on $\mathcal{P}$ that approximates that of a mixing system. In particular, initially localised Liouville densities should relax close towards the microcanonical measure at an exponential rate, on average. In this section we demonstrate that these signatures of chaos are exhibited also by the quantum dynamics. Most striking is the degree of similarity between the quantum and classical behaviours even in regimes with classically mixed dynamics.

We are interested in the behaviour of quantum probability distributions that are associated with measurements of classical dynamical variables. The quantum probability distribution associated with the classical observable $L_{z}$ is given by,

$$
P_{L_{z}}\left(m_{l}\right)=\left\langle\psi(n)\left|R_{l, m_{l}}\right| \psi(n)\right\rangle=\operatorname{Tr}\left[\left|l, m_{l}\right\rangle\left\langle l, m_{l}\right| \rho^{(l)}(n)\right]
$$


where,

$$
R_{l, m_{l}}=1_{s} \otimes\left|l, m_{l}\right\rangle\left\langle l, m_{l}\right|
$$

is a projection operator onto the eigenstates of $L_{z}$, and

$$
\rho^{(l)}(n)=\operatorname{Tr}^{(s)}[|\psi(n)\rangle\langle\psi(n)|],
$$

is the reduced state operator for the spin $\mathbf{L}$ at time $n$ and $\operatorname{Tr}^{(s)}$ denotes a trace over the factor space $\mathcal{H}_{s}$. We have written out the explicit expression (17) to emphasize that the probability of obtaining each $m_{l}$ value is associated with a projector onto a subspace of the factor space $\mathcal{H}_{l}$.

For reasons related to this fact (which we will make clear in later sections), we are also interested in examining the probability distributions associated with components of the total angular momentum $\mathbf{J}=\mathbf{S}+\mathbf{L}$. The probability of obtaining a given $m_{j}$ value upon measurement of $J_{z}$ is given by,

$$
P_{J_{z}}\left(m_{j}\right)=\sum_{m_{s}}\left|\left\langle\psi(n) \mid s, l, m_{s}, m_{j}-m_{s}\right\rangle\right|^{2}
$$

where $\left|s, l, m_{s}, m_{j}-m_{s}\right\rangle$ is an element of the orthonormal basis (2). The probability $P_{J_{z}}\left(m_{j}\right)$ is associated with a projector onto a subspace of the full Hilbert space $\mathcal{H}$. The dimension of each subspace is given by the number of pairs $\left(m_{s}, m_{l}\right)$ that yield a given value of $m_{j}=m_{s}+m_{l}$.

The classical probability distributions associated with dynamical variables are obtained by partial integration over the accessible phase space. In the case of $L_{z}$, the continuous marginal distribution is given by,

$$
P\left(L_{z}\right)=\iiint d S_{z} d \phi_{s} d \phi_{l} \rho_{c}\left(S_{z}, \phi_{s}, L_{z}, \phi_{l}\right)
$$

where for notational convenience we have suppressed reference to the time-dependence. The marginal probability distribution for the total spin component $J_{z}$ is obtained by integration subject to the constraint $S_{z}+L_{z}=J_{z}$,

$$
P\left(J_{z}\right)=\iiint \int d S_{z} d \phi_{s} d L_{z} d \phi_{l} \rho_{c}\left(S_{z}, \phi_{s}, L_{z}, \phi_{l}\right) \delta\left(S_{z}+L_{z}-J_{z}\right) .
$$

These classical distributions are continuous, though their quantum counter-parts are intrinsically discrete. To construct a meaningful quantum-classical comparison it is useful to discretize the classical distrbutions by integrating the continuous probabilities over intervals of width $\hbar=1$ centered on the quantum eigenvalues. In the case of the component $L_{z}$, the quantum probability $P_{L_{z}}\left(m_{l}\right)$ is then associated with the classical probability of finding $L_{z}$ in the interval $\left[m_{l}-1 / 2, m_{l}+1 / 2\right]$. This is given by

$$
P_{L_{z}}^{c}\left(m_{l}\right)=\int_{m_{l}-1 / 2}^{m_{l}+1 / 2} P\left(L_{z}\right) .
$$

Similarly, in the case of $J_{z}$, we compare each quantum $P_{J_{z}}\left(m_{j}\right)$ with the discrete classical probability,

$$
P_{J_{z}}^{c}\left(m_{j}\right)=\int_{m_{j}-1 / 2}^{m_{j}+1 / 2} P\left(J_{z}\right)
$$

In the following discussion of the numerical results we will emphasize that, for chaotic states, the steady-state shape of the quantum and classical distributions should be compared with the corresponding set derived from the microcanonical state. Our model is non-autonomous, but the spin magnitudes are conserved. The appropriate classical microcanonical measure is a constant on the accessible manifold $\mathcal{P}=S^{2} \times S^{2}$. This follows from the usual equilibrium hypothesis that all accessible microstates are equiprobable, where equiprobability is defined with respect to the invariant measure (8). This microcanonical density projected onto the $L_{z}$-axis produces the discrete, flat distribution,

$$
P_{L_{z}}^{m c}\left(m_{l}\right)=(2 l+1)^{-1} .
$$

However, projected along $J_{z}$, the microcanonical distribution is not flat, but has a tent-shape, 


$$
\begin{aligned}
P_{J_{z}}^{m c}\left(m_{j}\right) & =\frac{l+s+1-\left|m_{j}\right|}{(2 s+1)(2 l+1)} \text { for }\left|m_{j}\right| \geq l-s \\
& =\frac{1}{2 l+1} \text { for }\left|m_{j}\right| \leq l-s .
\end{aligned}
$$

In quantum mechanics, the equiprobability hypothesis implies that the appropriate microcanonical state is an equalweight mixture. This microcanonical state, sometimes called a random state, is proportional to the identity in the full Hilbert space $\mathcal{H}=\mathcal{H}_{s} \otimes \mathcal{H}_{l}$. It produces the same projected microcanonical distributions, i.e. (25) for $L_{z}$ and (26) for $J_{z}$, as the classical microcanonical state.

\section{A. Mixed Regime Chaos}

We consider first a classical parameter regime $(\gamma=1.215, r=1.1$, and $a=5)$ for which the kinematically accessible phase space $\mathcal{P}$ is highly mixed. The chaotic region appears to be connected (all chaotic initial conditions have the same largest Lyapunov exponent $\lambda_{L}=0.04$ ) and covers about half of the kinematic surface. A projection of only the chaotic initial conditions onto the plane spanned by $S_{z}$ and $L_{z}$ reveals large regular islands surrounding the stable parallel fixed points $\left( \pm S_{z}, \pm L_{z}\right)$, with chaotic regions spreading out from the unstable anti-parallel fixed points $\left( \pm S_{z}, \mp L_{z}\right)$. A similar projection of the regular initial conditions shows points not only clustered about the parallel fixed points but also spread along the axis $\tilde{S}_{z}=\tilde{L}_{z}$.

We now consider the time-evolution of quantum and classical states concentrated in the chaotic zone near one of the unstable anti-parallel fixed points, with initial centroids directed along $\theta(0)=\left(20^{\circ}, 40^{\circ}, 160^{\circ}, 130^{\circ}\right)$. The quantum

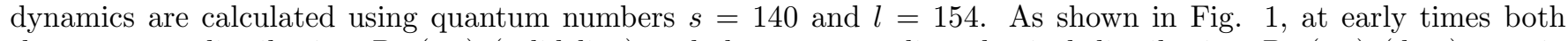
the quantum distribution $P_{L_{z}}\left(m_{l}\right)$ (solid line) and the corresponding classical distribution $P_{L_{z}}^{c}\left(m_{l}\right)$ (dots) remain well-localised. Their initial differences are not distinguishable on the scale of the figures. (The dots are shifted to the right by half of their width.) By time-step $n=20$ both quantum and classical distributions have broadened to the system dimension and begin to exhibit noticeable differences. As shown in Fig. 2, around $n=100$ the distributions have begun to settle close to an equilibrium shape. In Fig. 3 the successive time steps $n=199$ and $n=200$ show that, although both the quantum and classical distributions have relaxed very close to the same equilibrium distribution, the quantum distribution exhibits rapidly oscillating fluctuations about the classical steady-state.

Both the quantum and classical equilibrium distributions (projected along $L_{z}$ ) show significant deviation from the microcanonical distribution (25). This is also true of the distribution projected along $L_{x}$, which has a different nonuniform equilibrium distribution than that observed when projecting onto $L_{z}$ (see the left box of Fig. (1). Uniform marginal distributions would be expected if the classical mapping was mixing, in which case arbitrary initial densities (with non-vanishing measure) would relax to the microcanonical distribution. Since the accessible kinematic surface has large KAM surfaces in this parameter regime, the coarse-grained classical equilibrium distributions are not expected to be flat. An unexpected feature of the results is the observation that the shape of the equilibrium quantum distributions so accurately reflects the details of the KAM structure in the classical phase space. This feature is most striking in the case of the distributions projected along $J_{z}$ (see the right box in Fig. (1). The steady-state quantum and classical probability distributions $P_{J_{z}}\left(m_{j}\right)$ and $P_{J_{z}}^{c}\left(m_{j}\right)$ are both sharply peaked about $m_{j}=0$. This equilibrium shape is much more sharply peaked than the tent-shape of the projected microcanonical distribution, $P_{J_{z}}^{m c}\left(m_{j}\right)$, given by (26) and also plotted in the right box in Fig. 4 . The important point is that the additional localization of the quantum distribution can be understood from a standard fixed-point analysis of the classical map [13: the presence of KAM surfaces arising due to the stability of the parallel fixed points prevents the chaotic classical spins from aligning in parallel along the $z$-axis. Most remarkably, we find that the steady-state quantum distributions accurately reproduce this parameter-dependent structure of the mixed classical phase space even for much smaller quantum numbers. We examine how the accuracy of this correspondence scales with the quantum numbers in section VII.

\section{B. Regime of Global Chaos}

If we hold $a=5$ and $r=1.1$ fixed and increase the coupling strength to the value $\gamma=2.835$, then all four of the fixed points mentioned above become unstable [13]. Under these conditions less than $0.1 \%$ of the surface $\mathcal{P}$ is covered with regular islands; the remainder of the surface produces a connected chaotic zone with largest Lyapunov exponent $\lambda_{L}=0.45$. We will sometimes refer to this parameter regime as one of global chaos since the kinematically accessible phase space is predominantly chaotic.

The dynamics of the classical and quantum distributions are much simpler in this regime. We find that initially localised distributions, launched from arbitrary initial conditions, relax to the microcanonical distribution on a very 
short time-scale. To demonstrate this, we consider the dynamics of an initial quantum state with $s=140$ and $l=154$, and a corresponding classical density, launched from $\theta(0)=\left(20^{\circ}, 40^{\circ}, 160^{\circ}, 130^{\circ}\right)$. Though the initial distributions are the same as in the mixed regime, by time-step $n \simeq 6$ the quantum and classical distributions have already spread to the system dimension and begin to exhibit noticeable differences. By time-step $n \simeq 12$ both distributions have relaxed very close to the microcanonical distributions. We plot the equilibrium quantum and classical projected distributions $P_{L_{z}}\left(m_{j}\right)$ and $P_{J_{z}}\left(m_{j}\right)$ in Fig. 5 for time-step $n=50$. The projected classical distributions are early indistinguishable from the microcanonical forms, $P_{L_{z}}^{m c}\left(m_{j}\right)$ and $P_{L_{z}}^{m c}\left(m_{j}\right)$, and the quantum distributions again exhibit small fluctuations about the classical distributions. We have found that these equilibrium quantum-classical differences asymptote to a non-vanishing minimum when the measure of KAM surfaces becomes negligible. These minimum quantum fluctuations reflect characteristic deviations from the microcanonical state that arise because the equilibrium quantum state is pure, whereas the microcanonical state corresponds to a random mixture.

\section{RATES OF RELAXATION TO EQUILIBRIUM}

In order to characterize the time-scale of relaxation to equilibrium it is convenient to study the time-dependence of a scalar measure that is sensitive to deviations from the equilibrium state. A conventional indicator of this rate of approach to equilibrium is the coarse-grained entropy,

$$
H=-\sum_{i} P_{i} \ln P_{i} .
$$

Here the $\left\{P_{i}\right\}$ stand for the quantum probabilities associated with projectors onto some basis of microstates (e.g. the projected distributions discussed in the previous section). The sum (27) is a standard measure of the information contained in a probability distribution and is sometimes called the Shannon entropy.

The Shannon entropy has a number of useful properties. First, unlike the von Neumann entropy Tr $[\rho \ln \rho]$, the Shannon entropy is basis-dependent. It reduces to the von Neumann entropy if the 'chosen' basis diagonalizes the state operator. However, this basis, or, more precisely, the set of projectors onto the (time-dependent) spectral decomposition of the state operator, does not necessarily correspond to a set of classically meaningful observables. Our main interest is to examine correspondence at the level of classical dynamical variables, so we consider probabilty distributions associated with projectors onto the eigenstates of classically well-defined operators. The classical counterparts to these probability distributions are associated with some fixed partioning of the phase space into cells of width $\hbar$ along the axes of the associated dynamical variable.

Second, whereas the von Neuman entropy of the total system is constant in time $(\operatorname{Tr}[\rho \ln \rho]=0$ since $\rho=|\psi\rangle\langle\psi|)$, the basis-dependent Shannon entropy may have time-dependence even if the quantum state is pure. Thus (27) may be applied to examine the rate of relaxation of either pure or mixed quantum states. It is in this sense that we use the term relaxation, although the time-evolution is unitary in the quantum model (and volume-preserving in the classical model).

Given some fixed partioning of the phase space, if a classical state remains evenly spread through the phase space cells it occupies, and spreads through the phase-space exponentially with time, then an entropy like (27) should grow linearly with time. In this section we show that this argument holds approximately also for quantum states launched from a classically chaotic region of phase space. The actual rate of relaxation of the quantum states is accurately predicted by the classical entropy even for small quantum numbers.

We demonstrate this behaviour by first considering the quantum entropy $H_{q}\left[J_{z}\right]$ of the probabilities associated with the eigenvalues $m_{j}$ of $J_{z}$, i.e. the probabilities defined in (20). The corresponding classical entropy, $H_{c}\left[J_{z}\right]$, is calculated using the discrete classical probabilities (24). In Fig. 6 we compare the time-development of the quantum and classical entropies using quantum numbers $s=140$ and $l=154$. For these quantum numbers, the microcanonical (i.e. maximum) value of the entropy is $H^{m c}\left[J_{z}\right]=6.2$. In case $(\mathrm{c})$, corresponding to a regular zone of the mixed regime $\left(\theta(0)=\left(5^{\circ}, 5^{\circ}, 5^{\circ}, 5^{\circ}\right), \gamma=1.215\right)$, we actually see the greatest amount of difference between the quantum $\left(H_{q}\left[J_{z}\right]\right)$ and classical $\left(H_{c}\left[J_{z}\right]\right)$ entropies. $H_{q}$ exhibits a quasi-periodic oscillation about its initial value whereas for $H_{c}$ these oscillations eventually dampen. For smaller quantum numbers, and thus broader initial states, $H_{c}$ dampens much more rapidly although $H_{q}$ continues to exhibit a pronounced quasi-periodic behaviour. In case (b), with initial centroid $\theta(0)=\left(20^{\circ}, 40^{\circ}, 160^{\circ}, 130^{\circ}\right)$ set in a chaotic region of the equally mixed regime, both $H_{q}$ and $H_{c}$ oscillate about an initially increasing average before relaxing towards a constant value that lies well below the microcanonical maximum $H^{m c}\left[J_{z}\right]=6.2$. This saturation away from the maximum is expected in the classical model since a large fraction of the kinematic surface is covered with regular islands and remains inaccessible. In case (a), corresponding to the regime of global chaos $(\gamma=2.835)$ and with the same initial state as $(b)$, the entropies are nearly identical. Both 
grow much more quickly than in the mixed regime case, roughly linearly, until saturating very near the maximum value.

The quantum entropy is very well approximated by its classical counterpart also for smaller quantum numbers. In Fig. 7 we display the growth rates of the quantum and classical entropies of the probabilty distributions associated with the observable $L_{z}$ for three sizes of quantum system $(l=11, l=22, l=220)$ using the same parameters and initial condition as for data-set (a) in Fig. 6. In each case the quantum entropy is essentially identical to the corresponding classical entropy. The initial rate of growth is similar in each case, roughly linear, and of order the Lyapunov exponent, $\lambda_{L}=0.45$.

These results extend previous work demonstrating the that the widths of quantum states grow exponentially with time, on average, until saturation at the system dimension 110,12,13. Modulo the small quantum fluctuations, for both quantum and classical models we find that the subsequent relaxation to an equilibrium configuration occurs on the time-scale,

$$
t_{\text {rel }} \sim t_{\text {sat }}+\mathcal{O}\left(\lambda_{L}^{-1}\right)
$$

where $t_{s a t} \simeq \lambda_{w}^{-1} \ln l$ estimates the time it takes the initial coherent state to reach the system dimension. The exponent $\lambda_{w}$ is the exponent governing the growth rate of the state width [13]. The last term $\mathcal{O}\left(\lambda_{L}^{-1}\right)$ approximates the additional time-required for the state to become more or less uniformly spread over the accessible phase space. In predominantly chaotic regimes we have found that $\lambda_{w} \simeq \lambda_{L}$, though in mixed regimes $\lambda_{w}$ is generally a few times larger than the largest Lyapunov exponent.

\section{TIME-DEPENDENCE OF QUANTUM-CLASSICAL DIFFERENCES}

Before examing the scaling of quantum-classical differences with increasing quantum numbers, it is useful to determine first their time-domain characteristics under the different types of classical behviour. Previous work has shown that quantum-classical differences for low-order moments, though initially small, grow exponentially with time when the classical motion is chaotic [12,19] until the states approach the system size [13]. On this saturation time-scale those quantum-classical differences reach their maximum magnitude, but surprisingly this maximum was small, $\mathcal{O}(\hbar)$. More specifically, it did not scale with the quantum numbers. Of course, two distributions can be altogether different even when the differences between their means and variances are quite small, and therefore it is useful to examine the differences between the quantum and classical states in a more sensitive way.

In this section we examine the time-dependence of bin-wise deviations between the quantum and classical probability distributions. For the observable $L_{z}$ this indicator takes the form,

$$
\sigma\left[L_{z}\right]=\sqrt{\frac{1}{(2 l+1)} \sum_{m_{l}=-l}^{l}\left[P_{L_{z}}\left(m_{l}\right)-P_{L_{z}}^{c}\left(m_{l}\right)\right]^{2}}
$$

This standard deviation estimates typical quantum-classical differences on the scale of $\hbar$ along the $L_{z}$-axis. Each interval is centered on a quantum eigenvalue. The $P_{L_{z}}^{c}\left(m_{l}\right)$ correspond to a measurement, or coarse-graining, of the classical density on an extremely fine-scale.

In Fig. 8 we examine the time dependence of $\sigma\left[L_{z}\right]$ for the same three classical sets of parameters and initial conditions displayed in Fig. 6. The initial value of $\sigma\left[L_{z}\right]$ is generally not zero since it is not possible to match all the marginal distributions exactly in the case of the $\mathrm{SU}(2)$ coherent states [13]. The actual magnitude of the initial discrepancy depends on the angle between the axis of measurement, e.g. $L_{z}$, and the direction of polarization of the initial state. For both chaotic states the differences initially decrease from their angle-dependent value and then increase until saturation at a steady-state value. This steady-state value is reached much later in the mixed regime (upper solid line), than in the global chaos regime (lower solid line). It occurs on the time-scale, $t_{r e l}$, on which the underlying distributions have reached their steady-state configurations (modulo the quantum fluctuations).

As shown in Fig. 8, the quantum-classical differences are actually largest for the regular state (dotted line) of the mixed regime $(\gamma=1.215)$ at both early and late times (relative to the relaxation time-scale). The steadystate magnitude of the differences for the global chaos regime $(\gamma=2.835)$ is significantly smaller than the typical magnitude for the mixed regime. However, for larger values of the classical perturbation strength $\gamma$, this average steady-state magnitude does not decrease further (with the quantum numbers held fixed) but has reached a nonvanishing minimum. The magnitude of the minimum steady-state fluctuations, $D_{L_{z}} \simeq 2 \times 10^{-4}$, should be compared with a typical magnitude of the quantum and classical distributions, $P_{L_{z}}\left(m_{l}\right) \simeq 3 \times 10^{-3}$. In the following section we examine how these fluctuations scale with increasing quantum numbers. 
Above we have considered quantum-classical differences for observables (projectors onto subspaces) associated with the factor space $\mathcal{H}_{l}$. In this factor space the state is initially pure but becomes mixed as a result of dynamical interacions with the other subsystem. It is interesting to check if the dynamical behaviours of the differences are an artefact of this dynamical mixing. Therefore we consider also bin-wise quantum-classical differences for an observable $\left(J_{z}=S_{z}+L_{z}\right)$ that acts non-trivially on the full Hilbert space $\mathcal{H}=\mathcal{H}_{s} \otimes \mathcal{H}_{l}$. The quantum state in the full Hilbert space remains pure throughout the time-evolution. We construct the same standard deviation of the bin-wise differences between the quantum and classical probability distributions as above,

$$
\sigma\left[J_{z}\right]=\sqrt{\frac{1}{[2(s+l)+1)]} \sum_{m_{j}}\left[P_{J_{z}}\left(m_{j}\right)-P_{J_{z}}^{c}\left(m_{j}\right)\right]^{2}},
$$

where $m_{j} \in\{l+s, l+s-1, \ldots,-(l+s)\}$. In Fig. 9 we compare $\sigma\left[J_{z}\right]$ in the same three classical regimes examined in Fig. 8. Once again the regular state (dotted line) exhibits the largest quantum-classical differences, and the differences for both chaotic states (middle and lower solid line) grow to a steady-state value on the time-scale at which the underlying distributions relax to their equilibrium configurations. As above, the average value of the differences for $\gamma=2.835$ (lower solid line) correspond to a non-vanishing minimum, that is, the average value does not noticeably decrease for larger values of $\gamma$. The minimum quantum fluctuations are again small when compared with the average height of the probability distribution, $[2(s+l)+1]^{-1} \simeq 2 \times 10^{-3}$.

For $\gamma \simeq 2.835$, the measure of regular islands is already very close to zero and the classical system is nearly ergodic on $\mathcal{P}$. Similarly, the quantum state is no longer constrained by any invariant classical structures but spreads almost evenly about the accessible Hilbert space. We find that the standard deviations of the quantum fluctuations that account for the equilibrium quantum-classical differences approach a non-vanishing minimum as the classical dynamics approach ergodicity on $\mathcal{P}$. These equilibrium differences can not vanish (for fixed quantum numbers) because the total quantum state remains pure under the unitary dynamics, whereas the microcanonical equilibrium corresponds to an equal-weight mixture.

\section{CORRESPONDENCE IN THE CLASSICAL LIMIT}

We now turn to an examination of the classical limit, $\mathcal{J} / \hbar \rightarrow \infty$, where $\mathcal{J}$ is characteristic system action. Since the quantum-classical differences grow to their largest values once the states have spread to the system size and subsequently fluctuate about this magnitude, we will examine the scaling of the differences in this late time-domain, that is, when the states have relaxed close to their equilibrum configurations. Moreover, these scaling results will then complement previous work that has focussed on correspondence at early times [13], in the Ehrenfest regime when the states are narrow relative to the system dimensions.

We wish to determine if the standard deviation of the quantum-classical differences (defined in the previous section) decreases in magnitude with increasing quantum numbers. When comparing models with increasing quantum numbers, we hold the width of each probability bin fixed $($ at $\hbar=1)$. Since the number of bins will increase with the quantum number, it follows that the height of the probability distribution in a given bin will also decrease. Consequently, we construct a scale-independent, or relative, measure of the bin-wise quantum fluctuations by taking the ratio of the standard deviation to the average value of the probability distribution. For the observable $L_{z}$ this takes the form,

$$
R\left[L_{z}(n)\right]=\frac{D\left[L_{z}(n)\right]}{\bar{P}_{L_{z}}}=N_{l} \sigma\left[L_{z}(n)\right] .
$$

where the average value $\bar{P}_{L_{z}}=1 /(2 l+1)=1 / N_{l}$. If this relative measure approaches zero in the classical limit then the quantum probabilty distribution converges to the corresponding classical one in that limit.

In Fig. 10 we consider typical equilibrium values of $R\left[L_{z}(n)\right]$ plotted against $1 / \sqrt{N_{l}}$. We study the scaling using $N_{l}$ because it is equal to the dimension of the factor space $\mathcal{H}_{l}$ and it is also proportional to the subsystem size $N_{l} \simeq 2|\mathbf{L}|$. We first consider a state launched in the global chaos regime $(\gamma=2.835, r=1.1)$, with initial condition $\theta(0)=\left(45^{\circ}, 70^{\circ}, 135^{\circ}, 70^{\circ}\right)$. The scatter of plus signs for each $N_{l}=2 l+1$ value corresponds to time-steps $n$ such that $41 \leq n \leq 50$. These time-step values are chosen because they occur well after the relaxation time $t_{r e l} \simeq 6$. In this regime the data exhibits very little scatter. A least-squares fit to the curve $R=A / \sqrt{N_{l}}+B$ yields a value for the intercept $B$ that is consistent with zero $(B=0.001 \pm 0.001)$ and a slope of order unity $(A=1.032 \pm 0.02)$. An intercept consistent with zero implies that quantum-classical differences vanish in the classical limit, i.e. $P_{L_{z}}\left(m_{l}\right) \rightarrow P_{L_{z}}^{c}\left(m_{l}\right)$ as $l \rightarrow \infty$. This result is especially remarkable since we have considered the differences that arise given classical measurements which resolve the observable $L_{z}$ with the rather extraordinary precision of $\hbar=1$. 
We next consider a state launched from the chaotic zone of the mixed regime $(\gamma=1.215, r=1.1)$ with $\theta(0)=$ $\left(20^{\circ}, 40^{\circ}, 160^{\circ}, 130^{\circ}\right)$. The scatter of crosses in Fig. 10 corresponds to time-steps $191 \leq n \leq 200 n$, again chosen well after the relaxation time $t_{r e l}$ for the range of quantum numbers considered. The scatter of quantum-classical differences at each $N_{l}$ value is much more significant in this regime in which the equilibrium distributions reflect a much more complex phase space structure. However, the relative differences exhibit, on average, a similar dependence on the quantum numbers as in the predominantly chaotic regime. In this regime a least-squares fit to the curve $R=$ $A / N_{l}{ }^{1 / 2}+B$ yields a slope of order unity $(A=3.39 \pm 0.15)$ but a negative value for the intercept $(B=-0.017 \pm 0.009)$ within two-standard deviations of zero. A negative intercept is not physically meaningful (since $R$ is a positive definite quantity) and we assume it arises as a consequence of the statistical scatter in the data. Also plotted is the curve $R=C / N_{l}^{1 / 2}$, with slope $C=3.09 \pm 0.04$ also determined from a least-squares fit. Both fits are good, with reduced $\chi^{2}$ values of order unity.

As we noted in the last section, the subsystem states do not remain pure, because of dynamically induced entanglement between the subsystems. Since the subsystem state (19) in the factor space $\mathcal{H}_{l}$ is not pure, but highly mixed in the equilibrium time-domain, it is possible that the scaling with $N_{l}$ that we observe is related to the purity-loss from this entanglement. Consequently, it is useful to examine the scaling of the quantum-classical differences for the total spin $J_{z}$. The operator $J_{z}$ acts non-trivially in the full Hilbert space $\mathcal{H}$. In this Hilbert space the system is described by a pure state vector at all times. In Fig. 11 we consider the scaling of the ratio,

$$
R\left[J_{z}(n)\right]=\frac{D\left[J_{z}(n)\right]}{\bar{P}_{J_{z}}}=N_{j} \sigma\left[J_{z}(n)\right]
$$

where $\bar{P}_{J_{z}}=[2(s+l)+1]^{-1}=N_{j}^{-1}$ is the average value of either distribution, versus the dimension $N_{j}$. Here $N_{j}$ is the number of subspaces associated with distinct eigenvalues $\left(m_{j}\right)$ of the quantum operator $\left(J_{z}\right)$. In contrast with $N_{l}, N_{j}$ is not equal to the dimension of the corresponding Hilbert space, though it is a measure of the system size since $N_{j} \simeq 2|\mathbf{J}|$. The parameters and initial conditions shown in Fig. 11 are the same as in Fig. 10. The same fit procedure as above, but applied to the function $R=A / N_{j}^{1 / 2}+B$, yields a value for $B$ that is again consistent with zero $(B=0.00038 \pm 0.0016)$ and a positive slope of order unity $(A=2.00 \pm 0.04)$ in the predominantly chaotic regime (scatter of plus signs). Thus the relative standard deviation for $J_{z}$ also decreases as the square of the quantum numbers and fits to an intercept that is consistent with zero. This implies that the fluctuating quantum distributions approach the classical equilibrium, even for a few degree-of-freedom system, which is described at all times by a pure state. In a chaotic state of the mixed regime (scatter of crosses), the fluctuations are larger, and the same fit procedure as above gives $(B=-0.016 \pm 0.012, A=6.4 \pm 0.3)$, where the negative value for $B$ lies within two standard deviations of zero and is presumed to result from the statistical scatter of the data. Also plotted is the equation $R=C / N_{j}^{1 / 2}$, with $C=5.97 \pm 0.06$ determined from a least-squares fit. The fits to both equations are good, with reduced $\chi^{2}$ values of order unity.

\section{DISCUSSION}

We have shown that, in classically chaotic regimes, initially localised quantum states relax to an equilibrium configuration that reflects the details of the classical phase-space structure. We find a remarkable degree of correspondence between the quantum and classical relaxation rates, even for small quantum numbers. Moreover, contrary to results obtained for the low-order moments [12,13], the degree of difference between the probability distributions is actually smaller for the chaotic states than the regular states.

The equilibrium quantum distributions exhibit small rapidly oscillating fluctuations about the coarse-grained classical equilibrium. As the measure of regular islands on the classical manifold approaches zero, the quantum and classical equilibrium configurations approach their microcanonical forms, and the quantum fluctuations about the classical equilibrum approach a non-vanishing minimum. This minimum arises because we consider total quantum states that are pure, whereas the microcanonical configuration is produced by an equal-weight mixture.

For the distributions associated with the subsystem observable $\mathbf{L}$, the scale-independent standard deviation of these differences decreases as $N_{l}^{-1 / 2}$ where $N_{l}=2 l+1 \simeq 2|\mathbf{L}|$ is the dimension of the factor space, and becomes vanishingly small in the limit of large quantum numbers (i.e. large spins). These results suggest that correspondence with classical Liouville mechanics emerges in the classical limit for time-scales much longer than the Ehrenfest time.

A great deal of recent work has emphasized that the loss of purity resulting from interactions with a quantum environment removes characteristic quantum effects and improves the degree of quantum-classical correspondence [15.2,16]. While this is certainly the case for small quantum systems, it has been further argued that these decoherence 
effects must be taken into consideration to see the emergence of classical properties from quantum mechanics, even in the limit of large quantum numbers, if the classical motion is chaotic [5. 18 ].

Since our model is comprised of interacting subsystems, initially separable pure states become entangled dynamically; the subsystem states (in each factor space) do not remain pure but become mixed. This entanglement process has an effect that is analogous to the process of decoherence. Hence one might suspect that the emergent classical behaviour that we have observed for the properties of the subsystem $\mathbf{L}$ may be strictly the result of a "decoherence" effect arising from entanglement with the other subsystem. To address this possibility, we have considered also the quantum-classical differences that arise in the probability distributions for a total system observable, $J_{z}$. In this case the quantum observables are projectors onto subspaces of the full Hilbert space, rather than merely a factor space. The quantum state in this full Hilbert space is not subject to any entanglement or decoherence and remains pure throughout the unitary time-evolution. We have found that the scale-independent standard deviations for these quantum-classical differences decrease as $1 / \sqrt{N_{j}}$, where $N_{j}=2(s+l)+1$ is a measure of the system size, $N_{j} \simeq 2|\mathbf{J}|$. where $N=(2 s+1)(2 l+1)$ is the dimension of the Hilbert space. The bin-wise quantum-classical differences become increasingly difficult to observe, in the limit of large quantum numbers, even for system observables that are isolated

from the effects of decoherence. In this sense the process of decoherence is not necessary to produce quantum-classical correspondence in the classical limit.

\section{ACKNOWLEDGEMENTS}

We thank the Natural Sciences and Engineering Research Council of Canada for financial support. J. E. would

like to thank K. Kallio for stimulating discussions and the Centre for Experimental and Constructive Mathematics at Simon Fraser University for access to computational resources. 
[1] L.E. Ballentine, Y. Yang, and J.P. Zibin, Phys. Rev. A 50, 2854 (1994).

[2] S. Habib, K. Shizume and W.H. Zurek, Phys. Rev. Letters (1998).

[3] W.H. Zurek and J.P. Paz, Phys. Rev. Letters 72, 2508 (1994).

[4] G. Casati and B.V. Chirikov, Phys. Rev. Letters 75, 350 (1995).

[5] W.H. Zurek and J.P. Paz, Phys. Rev. Letters 75, 351 (1995).

[6] G.P. Berman and G.M. Zaslavsky, Physica 91A, 450 (1978).

[7] H. Frahm and H.J. Mikeska, Z. Phys. B 60, 117 (1985).

[8] F. Haake, M. Kus and R. Scharf, Z. Phys. B 65, 361 (1987).

[9] B.S. Helmkamp and D.A. Browne, Phys. Rev. E 49, 1831 (1994).

[10] R.F. Fox and T.C. Elston, Phys. Rev. E 50, 2553 (1994).

[11] R. Roncaglia, L. Bonci, B.J. West, and P. Grigolini, Phys. Rev. E 51, 5524 (1995).

[12] L.E. Ballentine and S.M. McRae, Phys. Rev. A 58, 1799 (1998).

[13] J. Emerson and L.E. Ballentine, in press in Phys. Rev. A. , quant-ph/0011020.

[14] D. Giulini et al. Decoherence and the Appearance of a Classical World in Quantum Theory (Springer, Berlin, 1996).

[15] B.S. Helmkamp and D.A. Browne, Phys. Rev. Lett. 76, 3691 (1996).

[16] J. Gong and P. Brumer, Phys. Rev. E. 60, 1643 (1999).

[17] W.H. Zurek and J.P. Paz, Physica D 83, 300 (1995).

[18] W.H. Zurek, Physica Scripta T76, 186 (1998).

[19] L.E. Ballentine, Phys. Rev. A. 63, 024101 (2000).

[20] A. Perelomov, Generalized Coherent States and Their Applications, (Springer-Verlag, New York, 1986).

[21] J.R. Dorfman, An Introduction to Chaos in Non-Equilibrium Statistical Mechanics (Cambridge University Press, Cambridge, 1999). 

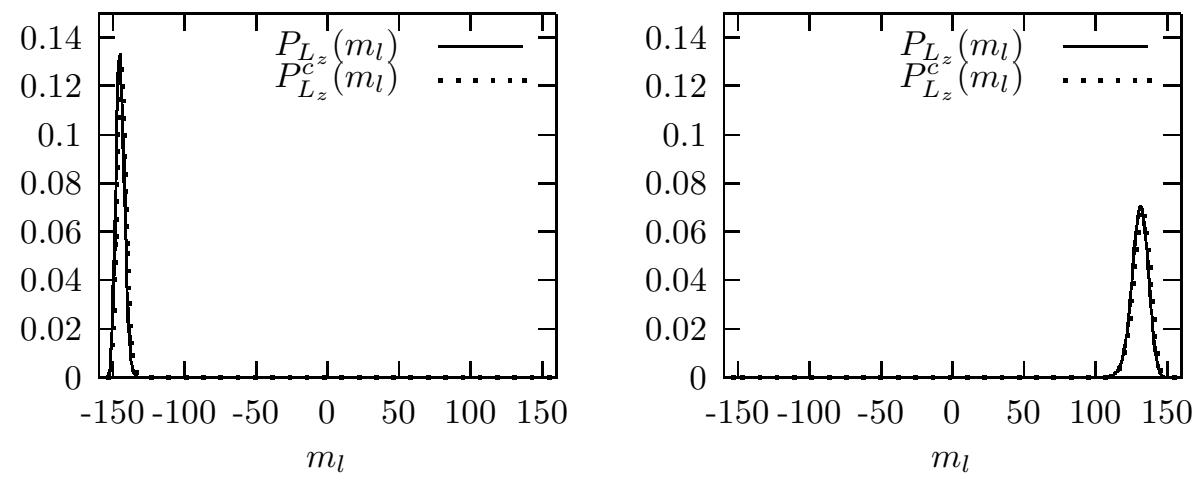

FIG. 1. Quantum and classical probability distributions for $L_{z}$ with $l=154$ in chaotic zone of mixed regime $(\gamma=1.215, r=1.1, a=5)$. The dots are visible because they are shifted to the right by half of their width. The figure on the left is the initial state $(n=0)$ and that on the right is at time-step $n=6$. 

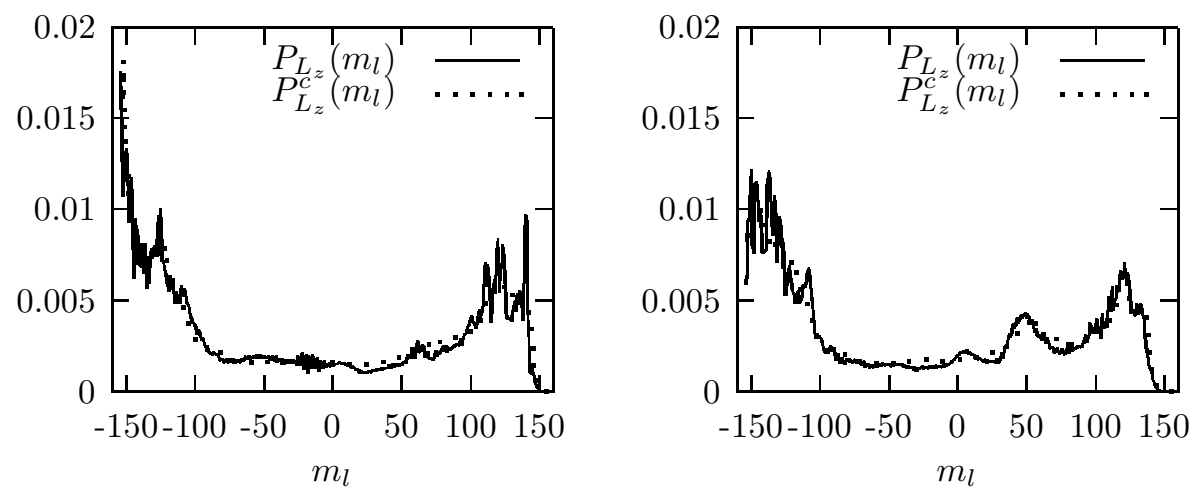

FIG. 2. Same as Fig. 1 but for time-steps $n=99$ on the left and $n=100$ on the right. Both quantum and classical distributions have reached the system dimension and are relaxing towards equilibrium. 

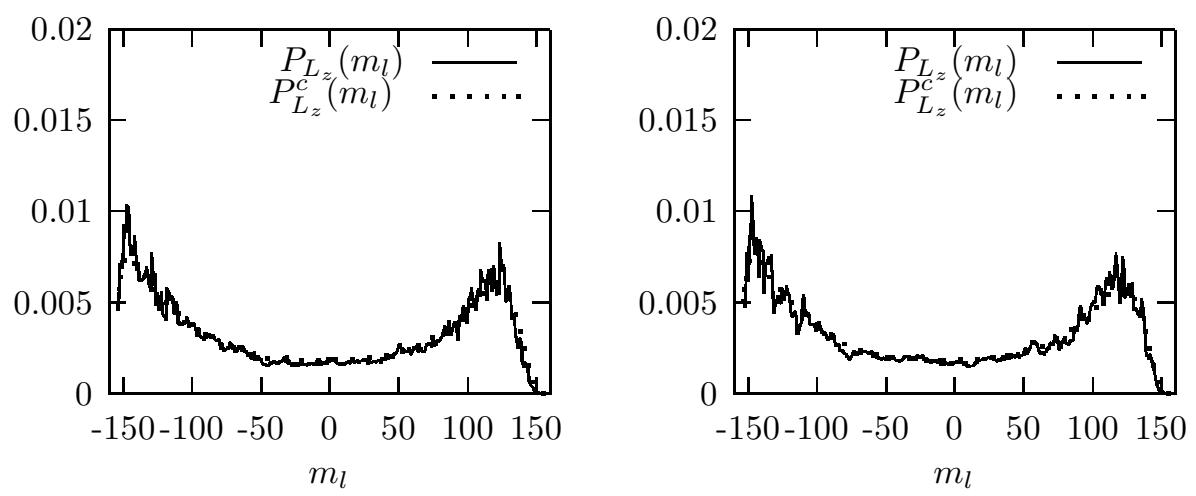

FIG. 3. Same as Fig. 1 but for $n=199$ on the left and $n=200$ on the right. The quantum distribution is fluctuating about a classical steady-state. 

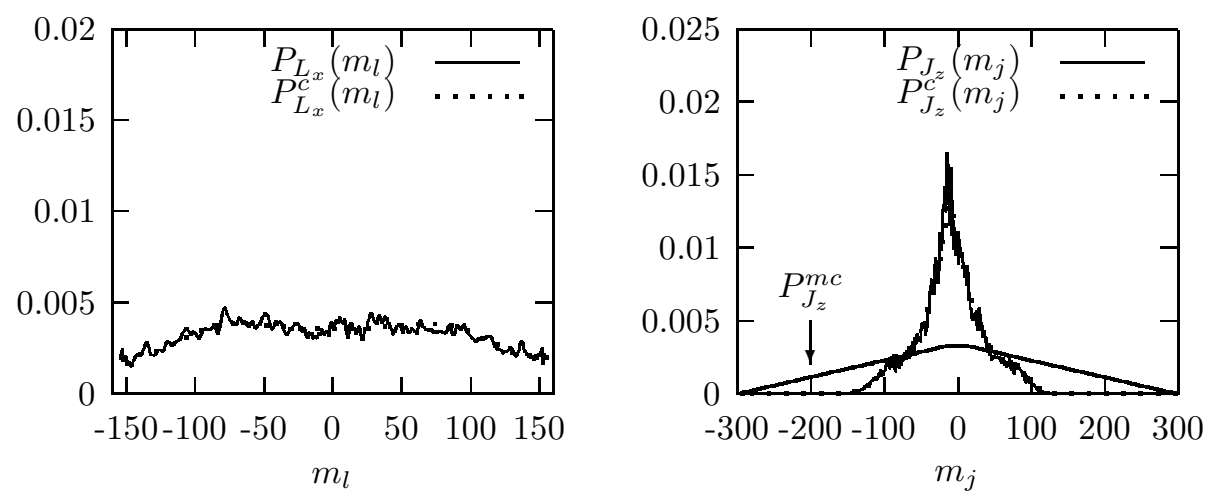

FIG. 4. Same as in the previous figure, but for $P_{L_{x}}\left(m_{l}\right)$ on the left and $P_{J_{z}}\left(m_{j}\right)$ on the right, at time-step $n=200$. Both $P_{J_{z}}\left(m_{j}\right)$ and $P_{J_{z}}^{c}\left(m_{j}\right)$ are localised relative to the projected microcanonical distribution $P_{J_{z}}^{m c}\left(m_{j}\right)$. 

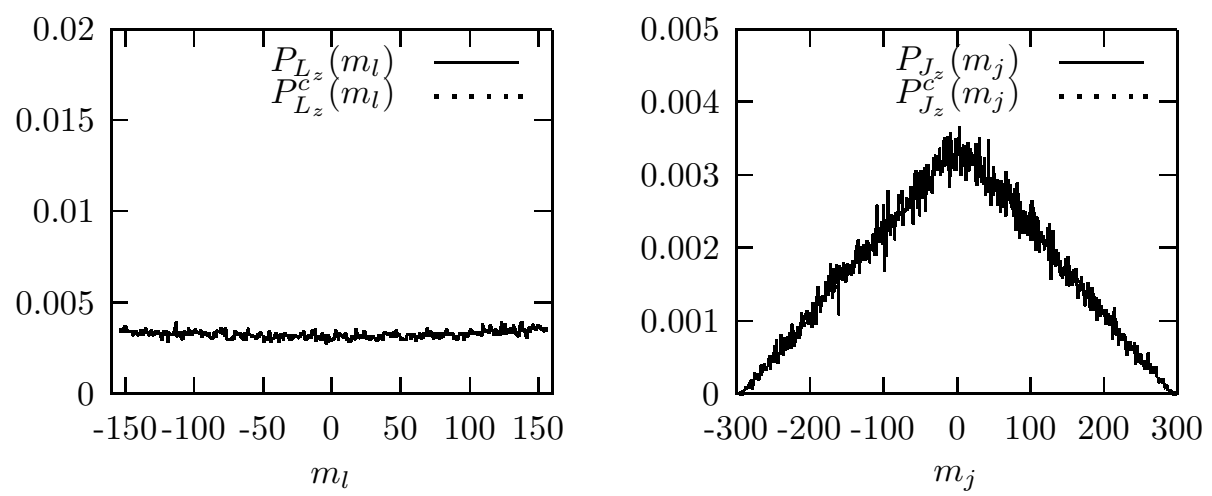

FIG. 5. The equilibrium shapes of $P_{L_{z}}\left(m_{l}\right)$ and $P_{J_{z}}\left(m_{j}\right)$ at time-step $n=50$ with $l=154$ for a state launched in the global chaos regime $(\gamma=2.835, r=1.1, a=5)$. The quantum distributions exhibit small rapidly oscillating fluctuations about the projected microcanonical distributions. The classical distributions are not visible since the points lie within the fluctuating quantum data. 


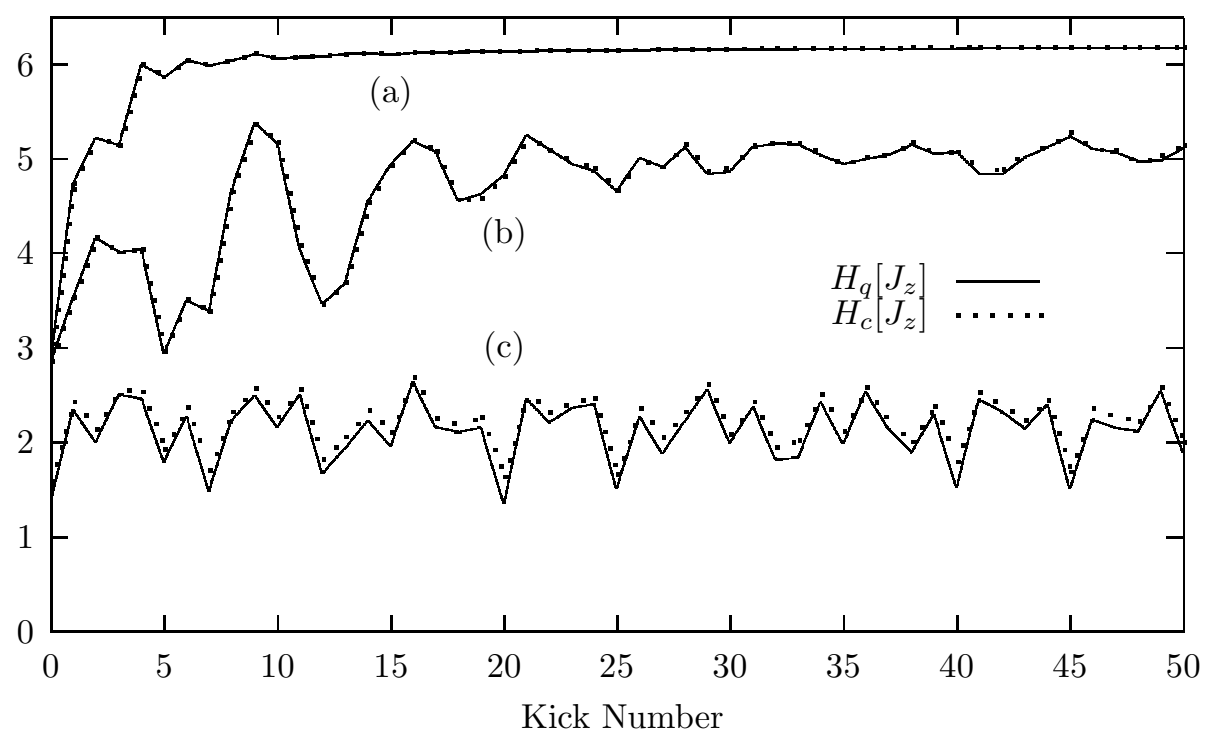

FIG. 6. Comparison of the quantum and classical entropies $H\left[J_{z}\right]=-\sum_{m_{j}} P_{J_{z}}\left(m_{j}\right) \log P_{J_{z}}\left(m_{j}\right)$ for $s=140$ and $l=154$ in (a) regime of global chaos $(\gamma=2.835)$; (b) chaotic zone of the mixed regime $(\gamma=1.215)$; (c) regular zone of the mixed regime $(\gamma=1.215)$. 


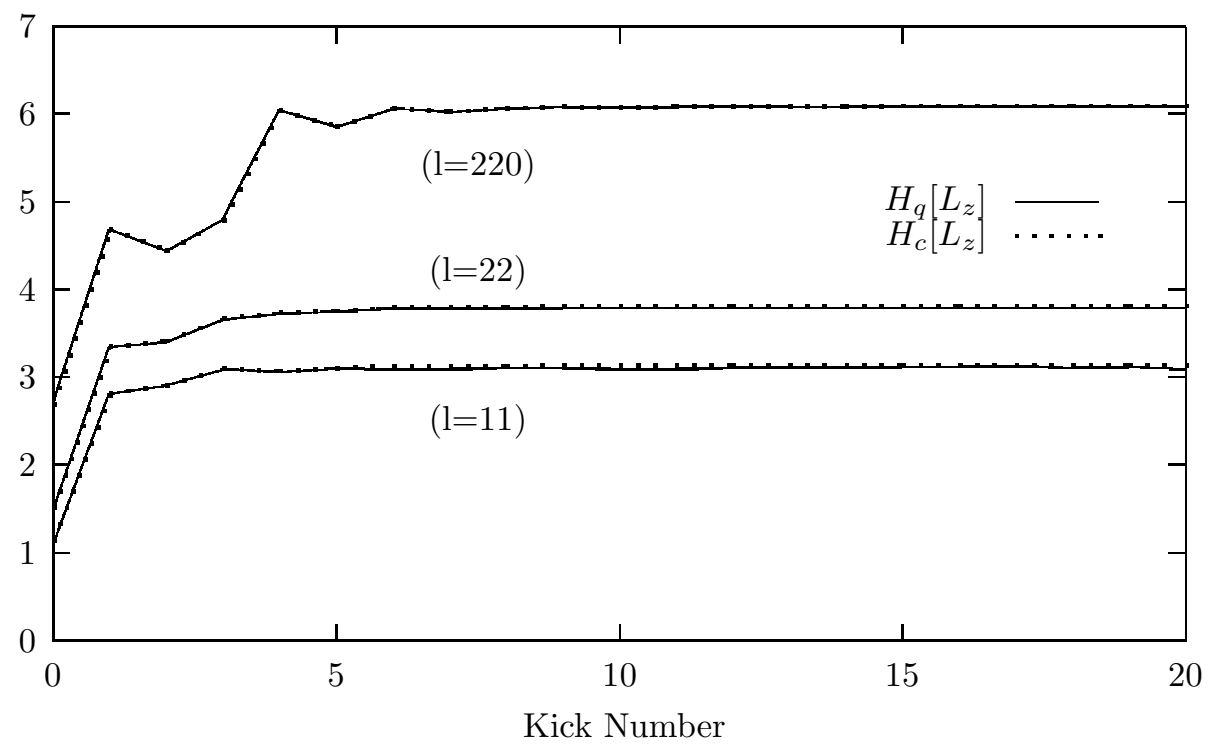

FIG. 7. Comparison of the quantum and classical subsystem entropies $H\left[L_{z}\right]=-\sum_{m_{j}} P_{L_{z}}\left(m_{l}\right) \ln P_{L_{z}}\left(m_{l}\right)$ for increasing system sizes in the global chaos regime of Fig. 6 . 


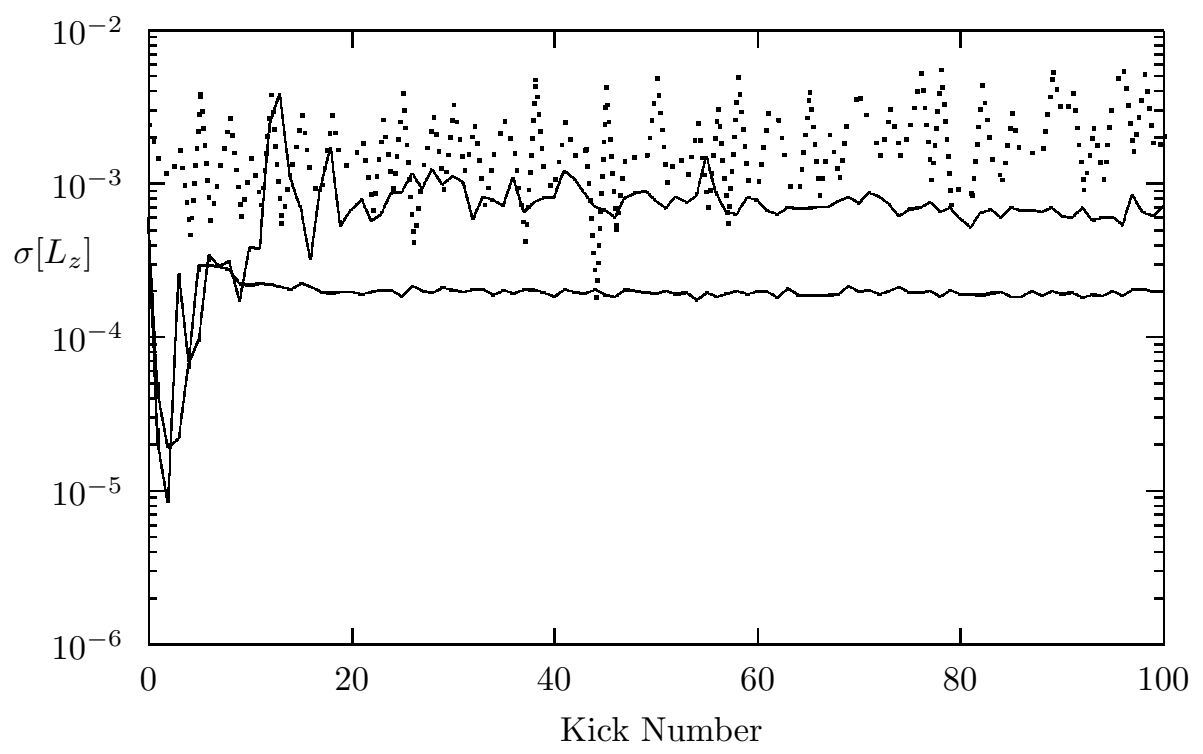

FIG. 8. Time dependence of the standard deviation $\sigma\left[L_{z}\right]$ of quantum-classical differences (29) for states launched from a regular zone (dotted line) of the mixed regime $(\gamma=1.215)$, from a chaotic zone of the same mixed regime (middle solid line), and from the regime of global chaos (lower solid line, $\gamma=2.835$ ). The initial discrepancy is relatively large, but quickly decreases, and then increases until reaching an asymptotic equilibrium value. This occurs more slowly for the mixed regime case, for which the asymptotic value is also larger. In all cases $s=140$ and $l=154$. 


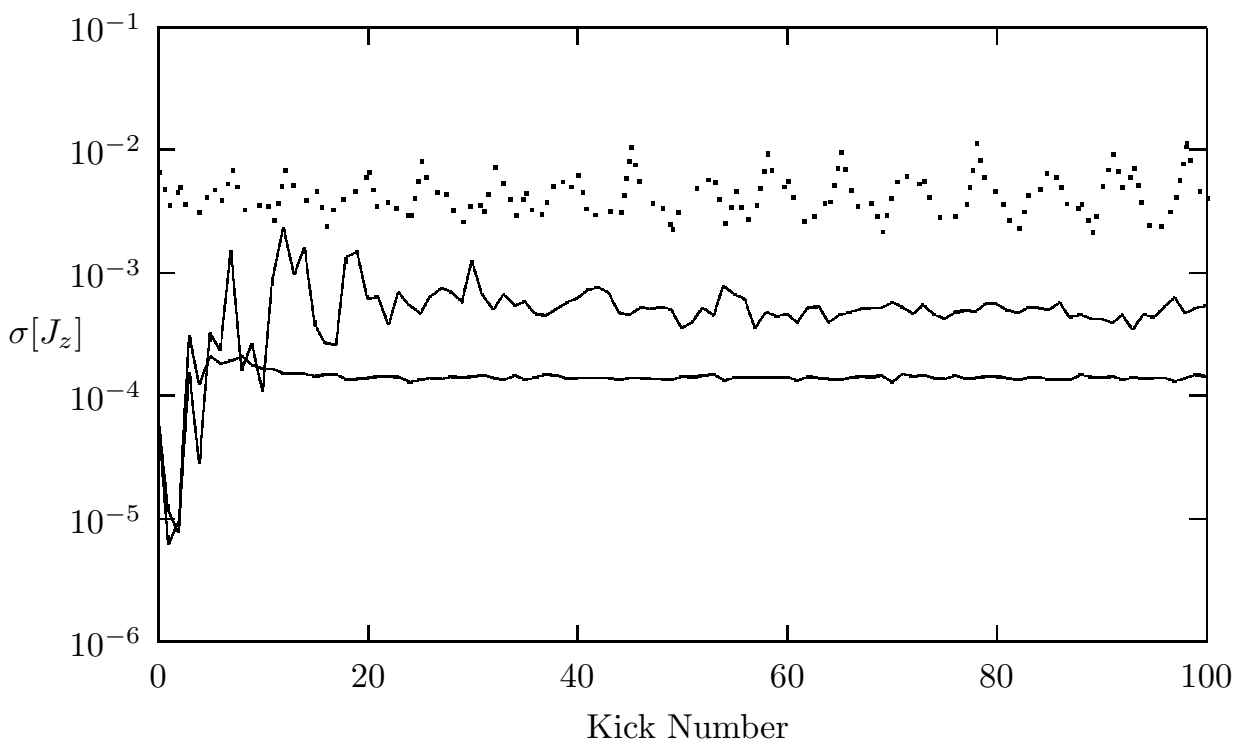

FIG. 9. Same as Fig. 月 but for the standard deviation of quantum-classical differences of the total angular momentum, $\sigma\left[J_{z}\right]$, given by $(30)$. 


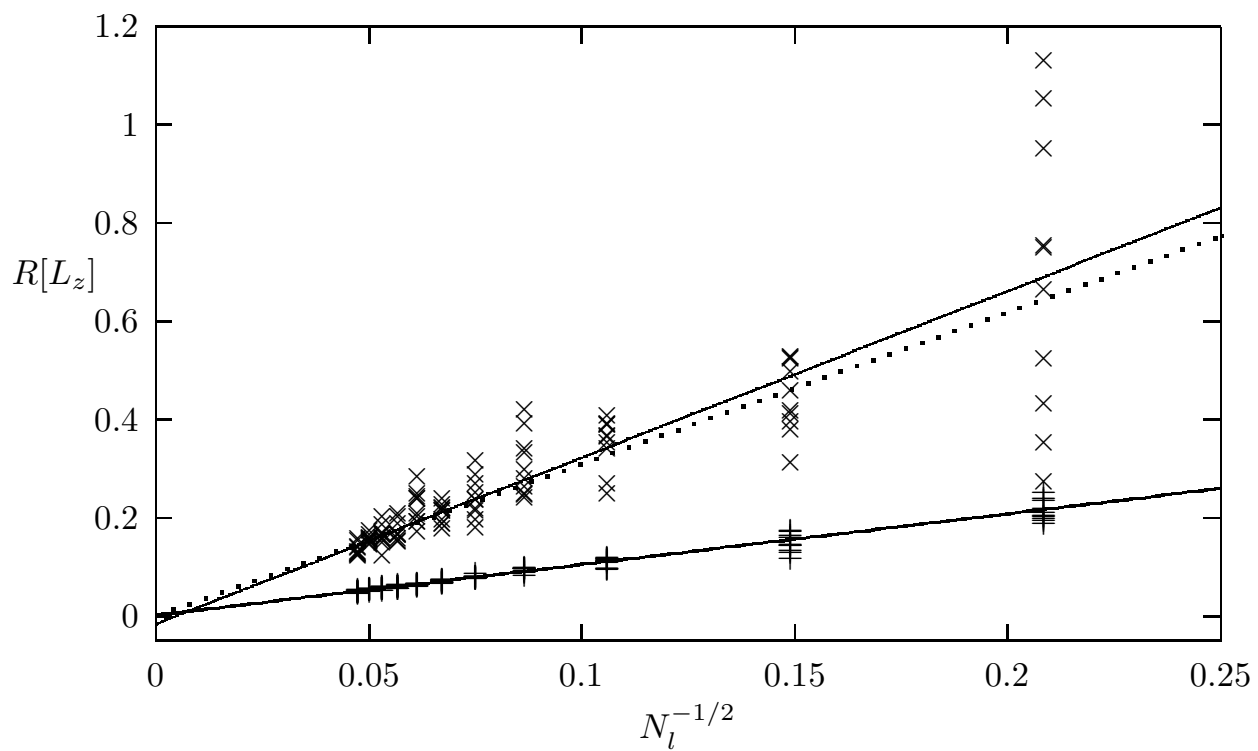

FIG. 10. Scaling of relative quantum-classical differences 31 in the equilibrum time-domain versus increasing system size. Scatter of crosses corresponds to time-steps $191 \leq n \leq 200$, for a state launched in the chaotic zone of the mixed regime $(\gamma=1.215)$. Scatter of plus signs corresponds to time-steps $41 \leq n \leq 50$, for a state launched in the global chaos regime. Data sets in both of these regimes are consistent with the scaling law $R \simeq N_{l}^{-1 / 2}$, where $N_{l}=2 l+1$. 


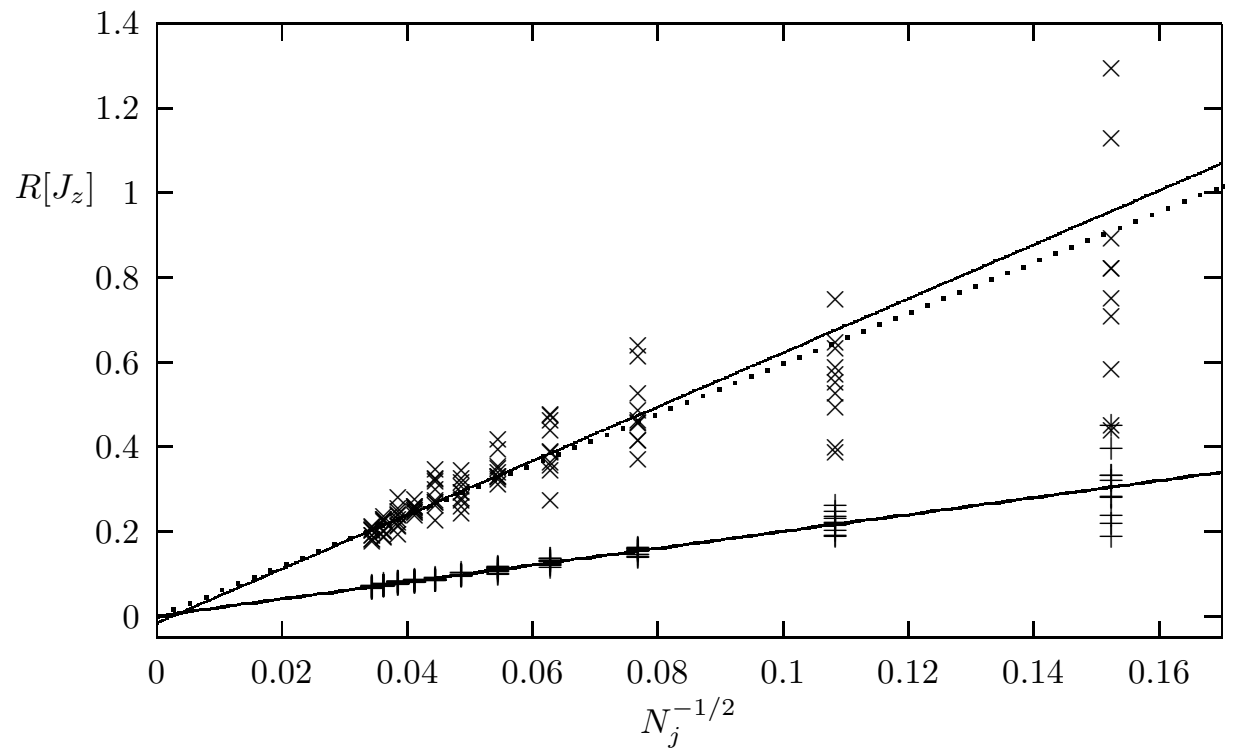

FIG. 11. Same as Fig. 10 but using $R\left[J_{z}\right]$, as given by (32). Data sets in both types of chaotic regime are consistent with the scaling law $R \simeq N_{j}^{-1 / 2}$, where $N_{j}=2(l+s)+1$. 